Hand (DASH) questionnaire and QuickDASH in people with rheumatoid arthritis BMC Musculoskeletal Disorders 2018; 19:118

[3] - Lima VP, Velloso M, Almeida FD, Carmona B, Ribeiro-Samora GA, Ferreira TJ. Testretest reliability of the unsupported upperlimb exercise test (UULEX) and 6-min peg board ring test (6PBRT) in healthy adult individuals.Physiotherapy Theory and Practice 2018; 34, 10:806812

[4] - Takahashi T, Jenkins SC, Strauss GR, Watson CP, Lake FR. A New Unsupported Upper Limb Exercise Test for Patients With Chronic Obstructive Pulmonary Disease. Journal of Cardiopulmonary Rehabilitation 2003;23:430-437.

Disclosure of Interests: None declared

DOI: 10.1136/annrheumdis-2019-eular.3919

\section{AB1384-HPR THE RELATIONSHIP BETWEEN QUALITY OF LIFE, PHYSICAL ACTIVITY LEVEL AND MENSTRUAL PAIN IN TURKISH WOMEN}

Gamze Nalan Çinar, Serap Özgül. Hacettepe University, Faculty of Health Sciences, Department of Physiotherapy and Rehabilitation, Ankara, Turkey

Background: Dysmenorrhea, defined as menstrual pain, is one of the common chronic pelvic pain problems associated with mussculoskeletal symptoms. ${ }^{1}$ Dysmenorrhea is considered as a lower abdominal or pelvic pain appears just before and/or during menstruation and lasts about 2448 hours. ${ }^{2}$ It is a common condition with prevalence rates between $50 \%$ and $91 \%$ in women. The daily life of women with menstrual pain can be adversely affected by pain and pain accompanying symptoms. ${ }^{3}$ It is seen that these effects are mostly on the quality of life (QoL) of individuals. In addition, physical activity of individuals may be restricted due to menstrual pain. ${ }^{4}$

Objectives: The aim of this study was to determine the relationship between quality of life, physical activity level and the severity of menstrual pain in Turkish women.

Methods: 336 female cases aged over 18 years and with menstrual pain complaint in the majority of menstrual cycles were included in the present study. Menstrual pain severity was assessed by $0-10$ point Visual Analogue Scale. ${ }^{5}$ Physical activity level was assessed by the "International Physical Activity Questionnaire-Short Form" and the score was expressed in Metabolic Equivalent Task minutes per week. Total score of the short form included walking, moderate level activity and duration (minutes) and frequency (days) of sufficiently active. The quality of life of was assessed by the "Nottingham Health Profile". It consists of six parts: pain, physical activity, energy, sleep, social isolation and emotional reaction. The maximum score on any section is 100 . The higher the score on any section the greater the number and severity of perceived problems in that area. Normality testing was performed on all data. Spearmans correlation was performed to identify correlation between menstrual pain score and other measures.

Results: The average age and body mass index of 336 participants were 223 years and $21,42,8 \mathrm{~kg} / \mathrm{m}^{2}$, respectively. There was a significant correlation between quality of life and menstrual pain severity $(r=0.350$; $\mathrm{p}: 0.006)$. There was no significant correlation between physical activity level and menstrual pain severity ( $p>0.05)$.

Conclusion: Based on the results of the present study, quality of life seems to be related with menstrual pain. On the other hand, no relationship was identified between the physical activity level and menstrual pain. In order to determine the exact relationship, further studies with larger samples and with more varied levels of physical activity are needed.

\section{REFERENCES}

[1] Kaya, S., Hermans, L., Willems, T., Roussel, N., \& Meeus, M. (2013). Central sensitization in urogynecological chronic pelvic pain: a systematic literature review. Pain Physician, 16(4), 291-308.

[2] Orhan, C., elenay, Ş. T., Demirtrk, F., zgl, S., zelpasacı, E., \& Akbayrak, T. (2018). Effects of menstrual pain on the academic performance and participation in sports and social activities in Turkish university students with primary dysmenorrhea: A case control study. Journal of Obstetrics and Gynaecology Research, 44(11), 2101-2109.

[3] Hillen, T. I., Grbavac, S. L., Johnston, P. J., Straton, J. A., \& Keogh, J. M. (1999). Primary dysmenorrhea in young Western Australian women: prevalence, impact, and knowledge of treatment. Journal of adolescent health, 25(1), 40-45.

[4] zgl S, zelpasacı E, Orhan C, Baran E, Beksa MS, Akbayrak T. Short-term effects of connective tissue manipulation in women with primary dysmenorrhea: A randomized controlled trial. Complementary Therapies in Clinical Practice. 2018;33:1-6.
[5] Wong C, Lai K, Tse H. Effects of SP6 acupressure on pain and menstrual distress in young women with dysmenorrhea. Complementary Therapies in Clinical Practice. 2010;16(2):64-9.

Disclosure of Interests: None declared

DOI: 10.1136/annrheumdis-2019-eular.6958

\section{AB1385-HPR TRUNK STRENGTH AND SPINAL MOBILITY IN SPONDYLOARTHRITIS PATIENTS}

Sophie De Mits ${ }^{1,2}$, Liselotte Deroo ${ }^{1,3}$, Ann-Sophie De Craemer ${ }^{1,3}$,

Thomas Renson ${ }^{1,3}$, Tine Willems $^{2}$, Lieven Danneels ${ }^{2}$, Philippe Carron ${ }^{1,3}$, Filip van den Bosch ${ }^{1,3}$, Dirk Elewaut ${ }^{1,3} .{ }^{1}$ Ghent University Hospital, Rheumatology, Ghent, Belgium; ${ }^{2}$ Ghent University, Rehabilitation Sciences, Ghent, Belgium; ${ }^{3}$ Ghent University, VIB Inflammation Research Center, Ghent, Belgium

Background: Spondyloartritis $(\mathrm{SpA})$ can seriously affect spinal mobility and trunk strength. Even though exercise therapy is considered one of the keystones of non-pharmacological treatment ${ }^{1}$, guidelines on exercise programs remain vague due to a lack of objective measurements of physical parameters such as trunk mobility and strength. Data on these parameters are scarce for both axial and peripheral SpA-patients (axSpA and perSpA pts).

Objectives: The aim of this study was to measure trunk strength and spinal mobility in SpA-pts and compare these parameters to healthy subjects matched for gender and age and to determine differences between the pts when grouped based on symptom duration and on presence of radiographic sacroilitis.

Methods: SpA-pts of the Be-Giant cohort were consecutively asked to participate in the study. After informed consent, BASDAI, BASFI and BASMI were evaluated. To measure trunk and cervical strength, pts performed 2 repetitions of a maximal isometric contraction for flexion, extension, lateral flexion and rotation on the David Back devices (DBD) after measuring the spinal mobility in these directions. The maximum value of the 2 repetitions was kept for further analysis. For assessments of lateral flexion and rotation, measured with the DBD, the mean was calculated for right and left measurements. Spinal mobility and trunk strength were compared with a healthy reference population, matched for gender and age by means of Wilcoxon singed-rank tests. When comparing the per$\mathrm{SpA}$ with the r-axSpA and the nr-axSpA, a Kruskal Wallis test was used. A Mann-Whitney $U$ test was used to check for differences between groups based on symptom duration.

Results: Thirty-one SpA-pts participated of which 18 were male (58\%). Twenty-four $(77 \%)$ were classified as axSpA and $7(23 \%)$ as perSpA. Six $(19 \%)$ of the axial pts had radiographic sacroilitis and 18 (58\%) were non-radiographic. Median time since diagnosis was 5 years and median symptom duration was 7.8 year. Mean age of the pts was 41 years (range: 2158 years) and their BMI was on average 24 (range: 17-33) Averages for BASDAI, BASFI and BASMI were 2.6 (range 0.0-6.0), 1.7 (range 0-6.8) and 0.9 (range 0.6-4.4) respectively.

SpA-pts showed decreased mobility for cervical flexion $(p<0.001)$, extension $(p<0.001)$ and rotation $(p<0.001)$ and trunk rotation $(p=0.001)$ compared to the healthy population. Cervical and trunk muscle strength was significantly decreased in SpA-pts in all directions compared to the reference population (flexion: $p=0.02$, other directions: $p<0.001$ ).

When comparing mobility and strength based on groups by radiographic axial or peripheral involvement, no significant differences could be detected.

When grouped based on symptom duration, the pts with longer standing symptoms ( $>7.8$ year) show a significant reduced mobility for cervical extension $(p=0.004)$ and rotation $(0.049)$ and lumbar rotation $(p=0.033)$ and a trend toward significance for lumbar extension $(\mathrm{p}=0.066)$ compared to those with shorter symptom duration ( $<7.8$ year). For strength no significant differences could be demonstrated between the groups.

Conclusion: Results of this study showed that SpA-pts have less mobility and decreased strength when compared with healthy gender- and agematched controls.

When comparing within the patient group, based on radiographic involvement, there were no differences neither for mobility or strength. Only discrete differences were found for mobility and no differences for strength when grouped based on symptom duration.

Therefore, rehabilitation of all SpA-pts should focus not only on mobility but should include trunk strengthening exercises as well.

\section{REFERENCES}

[1] van der Heijde D. et al. 2016 Ann Rheum Dis 2017;76;978-991 
Disclosure of Interests: Sophie De Mits: None declared, Liselotte Deroo: None declared, Ann-Sophie De Craemer: None declared, Thomas Renson: None declared, Tine Willems: None declared, Lieven Danneels: None declared, Philippe Carron: None declared, Filip van den Bosch Consultant for: AbbVie, BMS, Galapagos, Janssen, Lilly, Merck, Novartis, Pfizer and UCB, Speakers bureau: AbbVie, BMS, Janssen, Lilly, Merck, Novartis, Pfizer and UCB., Dirk Elewaut: None declared

DOI: 10.1136/annrheumdis-2019-eular.6868

\section{AB1386-HPR FORCE SENSE EVALUATION OF THE KNEE JOINT FOLLOWING MENISCECTOMY RELATED WITH DEGENERATIVE MENISCAL TEAR}

Cansu Gevrek ${ }^{1}$, Gizem Irem Kinikli', Ahmet Ozgur Atay ${ }^{2} .{ }^{1}$ Hacettepe University, Faculty of Physical Therapy and Rehabilitation, Ankara, Turkey; ${ }^{2}$ Hacettepe University Faculty of Medicine, Department of Orthopaedics and Traumatology, Ankara, Turkey

Background: Meniscal tears are associated with degenerative knee disease, which can range from mild chondral changes not visible on a radiograph to established knee osteoarthritis. Meniscectomy related with degenerative meniscal tear may affect the force sense of the knee joint which is defined as the ability to accurately reproduce a given force.

Objectives: The aim of this study was to compare the force sense of the knee joint of the patients with degenerative meniscal tear following meniscectomy with healthy peers.

Methods: Fifteen patients with meniscectomy and 18 healthy aged matched individuals were included in this study. Evaluation of force sense in the knee joint was measured with the use of a pressure biofeedback device, sphygmomanometer, (Stabilizer, TM, Chattanooga Group Inc., Chattanooga, TM). The pressure bag of this device was placed under the knee joint. All subjects were instructed to extend the knee via Maximal Voluntary Isometric Contraction (MVIC) for $5 \mathrm{~s}$. Each measurement was repeated three times with 1 -min rest intervals and the average constant errors observed on the pressure biofeedback device were recorded as $\mathrm{mmHg}$. Subjects were instructed to obtain at $50 \%$ of MVIC. They were asked to maintain the contraction (reproduce the target force) for $5 \mathrm{~s}$. The reproduction force was subtracted from the target force to create a trial error score. Higher error scores indicated lower force sense. Mann Whitney $U$ test was used for group comparisons. Statistical significance was defined as a $p$ value $<0.05$.

Results: There were no difference in terms of mean age, body mass index between patients $\left(53.67 .2\right.$ years, $\left.26.21 .8 \mathrm{~kg} / \mathrm{m}^{2}\right)$ and healthy peers $\left(53.47 .09\right.$ years, $\left.25.83 .2 \mathrm{~kg} / \mathrm{m}^{2}\right)$, respectively $(p>0.05)$. Lower accuracy of the knee extensors during the $50 \%$ MVIC task was significantly seen in patients with meniscectomy $(5.422 .07 \mathrm{mmHg})$ compared to healthy group $(1.881 .1 \mathrm{mmHg})(p<0.001)$

Conclusion: Results of this study demonstrated that higher force sense deficits are present in patients with meniscectomy related to degenerative meniscal tear compared to healthy peers. Thus, knee proprioceptive training focus on force sense might be developed to improve functional capabilities of knee joint especially following meniscectomy. It is also important to establish effective and feasible evaluation interventions for health professionals in clinical practice for assessing proprioception in terms of force sense in degenerative knees.

\section{REFERENCES}

[1] Sihvonen, R., Paavolah, M,D., Malmivaara, A., Itl, A., Joukainen, A., Nurmi, H., Halske, J., Jrvinen T.L.N. (2013). Arthroscopic Partial Meniscectomy versus Sham Surgery for a Degenerative Meniscal Tear. The new england journal of medicine, 369:2515-2524.

[2] Jarraya, M., Roemer, F.W., Englund, M., Crema, M.D., Gale, H.I., Hayashi, D., Katz J,N,. Guermazi A. (2017). Meniscus morphology: Does tear type matter? A narrative review with focus on relevance for osteoarthritis research. Seminars in Arthritis and Rheumatism 46:552561.

Disclosure of Interests: None declared

DOI: 10.1136/annrheumdis-2019-eular.3831

\section{AB1387-HPR ASPECTS OF VALIDITY AND RELIABILITY OF THE SWEDISH VERSION OF PATIENT HEALTH QUESTIONNAIRE-8 IN INDIVIDUALS WITH SYSTEMIC SCLEROSIS}

Malin Mattsson ${ }^{1}$, Gunnel Sandqvist ${ }^{2}$, Roger Hesselstrand ${ }^{2}$, Elisabet Dyrsmeds ${ }^{3}$, Annika Nordin ${ }^{4}$, Carina Boström ${ }^{1} .{ }^{1}$ Karolinska Institutet, Department of Neurobiology, Care sciences and Society, Stockholm, Sweden; ${ }^{2}$ Lund University, Department of Clinical Sciences, Lund, Sweden; ${ }^{3}$ Karolinska University Hospital, Unit of Rheumatology, Stockholm, Sweden; ${ }^{4}$ Karolinska Institutet, Department of Medicine, Stockholm, Sweden

Background: For assessing symptoms of depression in systemic sclerosis (SSc), the Patient Health Questionnaire-8 (PHQ-8) may be useful in clinical care as it is short and easy to administer. The English version of PHQ-8 has been found to be reliable and valid in SSc.

Objectives: To assess aspects of validity and reliability of PHQ-8 in Swedish (PHQ-8 Swe) for individuals with SSc.

Methods: Patients meeting the 2013 ACR/EULAR SSc criteria were recruited. The PHQ-8 Swe content validity was assessed via individual interviews (11 patients, 10 healthcare professionals, HPs) which was transcribed and analysed by content analysis. Patients ages, disease durations and symptoms of depression varied. The HPs had different occupational backgrounds and experiences in SSc care. Reliability was tested by internal consistency and test-retest reliability. Sixty-seven patients (median age 62 [minmax: 2887]) completed the PHQ-8 Swe on two different occasions.

Results: Content validity: The instruction, items, and response options were generally considered easy to understand; however, some clarifications were suggested. Among HPs, it was expressed that some items could be perceived as emotionally demanding for patients especially in recent onset disease. Further they experienced that PHQ-8 was problemrather than possibility-based. Introducing the PHQ-8 to patients and the need for follow-ups of the answers to the questionnaire were stated as essential. It was further expressed that PHQ-8 covered key aspects of symptoms of depression in SSc nevertheless examples of items suggested to be included were purpose in life, thoughts about death, and loneliness. The items were overall experienced as important without redundancy, and that some items could reflect more general SSc-related symptoms.

Cultural adaption: Possible adjustments to the PHQ-8 translation were made. This version was back translated into English and compared with the original English version. Thereafter, minor changes were made. A final PHQ-8 Swe version was tested for reliability.

Reliability: Cronbachs alpha was 0.86 and the corrected item-to-total correlation range was 0.420 .78 . There were no significant differences between testretest for seven of the eight items. The median weighted kappa coefficient was 0.63 . The median PHQ-8 Swe total score was 4 (020, no-severe depressive symptoms). There were no significant differences for total scores between test and retest and the ICC was 0.81 .

Conclusion: Content validity of PHQ-8 Swe was satisfactory and a positive quality was indicated for aspects of reliability in individuals with SSC tested in this study. Although some items could be interpreted as covering more general SSc-related symptoms, the PHQ-8 could be valuable for detecting patients with symptoms of depression in need of discussing emotional issues, as reflected in our study. Further studies of the PHQ-8 Swes usefulness are currently being undertaken via evaluation of other psychometric aspects.

\section{REFERENCES}

[1] -Kroenke K, et al. The PHQ-8 as a measure of current depression in the general population. J Affect Disord. 2009; 114:163-73

[2] -Milette K, et al. Comparison of the PHQ-9 and CES-D depression scales in systemic sclerosis: internal consistency reliability, convergent validity and clinical correlates. Rheumatology. 2010; 49:789-96

\section{ACKNOWLEDGEMENT}

Approval to adjust a translation of PHQ-8 to Swedish for patients with SSc was received from one of the original developers. We thank patient research partners Monica Holmnr and Astrid von Rosen for their valuable contribution. Funding was received from Region Norrbotten.

Disclosure of Interests: None declared

DOI: 10.1136/annrheumdis-2019-eular.4076 